\title{
UHPLC-ESI-OT-MS Phenolics Profiling, Free Radical Scavenging, Antibacterial and Nematicidal Activities of "Yellow-Brown Resins" from Larrea spp.
}

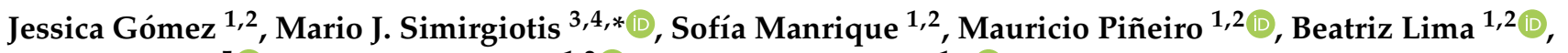

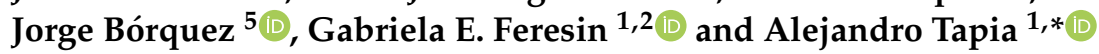

1 Instituto de Biotecnología-Instituto de Ciencias Básicas, Universidad Nacional de San Juan, Av. Libertador General San Martín 1109 (O), San Juan CP 5400, Argentina; jesicagomez674@gmail.com (J.G.); manriquesofia2@gmail.com (S.M.); mauridpg@gmail.com (M.P.); blima@unsj.edu.ar (B.L.); gferesin@unsj.edu.ar (G.E.F.)

2 CONICET (Consejo Nacional de Ciencia y Tecnología), CABA, Buenos Aires C1405DJR, Argentina

3 Instituto de Farmacia, Facultad de Ciencias, Campus Isla Teja, Universidad Austral de Chile, Valdivia 5090000, Chile

4 Center for Interdisciplinary Studies on the Nervous System (CISNe), Universidad Austral de Chile, Valdivia 5090000, Chile

5 Laboratorio de Productos Naturales Depto. de Química, Facultad de Ciencias, Universidad de Antofagasta, Av. Coloso S-N, Antofagasta 1240000, Chile; jorge.borquez@uantof.cl

* Correspondence: mario.simirgiotis@gmail.com (M.J.S.); atapia@unsj.edu.ar (A.T.); Tel.: +56-063-2244369 (M.J.S.); +54-264-4211700-294 (A.T.)

check for updates

Citation: Gómez, J.; Simirgiotis, M.J.; Manrique, S.; Piñeiro, M.; Lima, B.; Bórquez, J.; Feresin, G.E.; Tapia, A. UHPLC-ESI-OT-MS Phenolics Profiling, Free Radical Scavenging, Antibacterial and Nematicidal Activities of "Yellow-Brown Resins" from Larrea spp. Antioxidants 2021, 10, 185. https://doi.org/

10.3390/antiox10020185

Academic Editor: Filomena Nazzaro Received: 30 December 2020

Accepted: 22 January 2021

Published: 28 January 2021

Publisher's Note: MDPI stays neutral with regard to jurisdictional claims in published maps and institutional affiliations.

Copyright: (c) 2021 by the authors. Licensee MDPI, Basel, Switzerland This article is an open access article distributed under the terms and conditions of the Creative Commons Attribution (CC BY) license (https:// creativecommons.org/licenses/by/ $4.0 /)$.

\begin{abstract}
This research was designed to investigate the metabolite profiling, phenolics and flavonoids content and the potential antioxidant, antibacterial and nematicidal activities of "yellow-brown resins" from Larrea divaricata Cav (LdRe) and L. nitida Cav (LnRe). Metabolite profiling was obtained using an ultrahigh resolution liquid chromatography orbitrap MS analysis (UHPLC-ESI-OT-MS). The antioxidant properties were screened by four methods: 2,2-diphenyl-1-picrylhydrazyl assay (DPPH), trolox equivalent antioxidant activity assay (TEAC), ferric-reducing antioxidant power assay (FRAP) and lipid peroxidation in erythrocytes (LP). The antibacterial activity was evaluated according to the Clinical and Laboratory Standards Institute (CLSI) guidelines. In addition, the potential combinatory effect was analyzed with the fractional inhibitory concentration index (FICI) values using the checkerboard design. The nematicidal activity was carried out according to a standardized protocol. LdRe and LnRe showed a strong capture of the DPPH radical withvalues around $8.4 \mu \mathrm{g}$ resin/mL; FRAP (1.69-1.94 mgTE/ g resin), TEAC (1.08-1.09 mgTE/g resin) and LP (81-82\% at $100 \mu \mathrm{g}$ of resin/mL) assays. A strong antimicrobial activity was displayed by both resins against methicillin-sensitive Staphylococcus aureus ATCC 25923(MSSA) and methicillin-resistant S. aureus ATCC 43300(MRSA) (MICs $=16-32 \mu \mathrm{g}$ resin $/ \mathrm{mL}$ ). Additionally, the combination of LdRe or LnRe with the antibiotic cefotaxime showed an indifferent effect (FICI values $=1-1.25$ ), however, this combinationcould be a potential strategy to reduce the drug doses, and in this way can be a potential alternative to reduce bacterial resistance. On the other hand, the resins showed a scarce nematicidal potential toward J2 Meloidogyne incognita; an important nematode infecting horticultural crops. Phenolics compounds were identified by UHPLC-PDA-OT-MS analysis, updating the knowledge on the chemical profile of these species. These results, together with the high content of quantified phenolics and flavonoids, allow the phenolics-enriched resins of these two Larrea species to be considered as a promising sustainable source of compounds of pharmacological interest.
\end{abstract}

Keywords: dipping in dichloromethane; biomolecules of pharmacological interest; Larrea divaricata; L. nitida; lignans 


\section{Introduction}

The resins are naturally secreted by species of the families Asteraceae, Burseraceae, Fabaceae, Pinaceae and Zygophyllaceae. Their chemical profiles are characterized by phenolic compounds, terpenes, flavonoids, chalcones, lignans and fats, some of which display protective functions against natural predators and pathogens of these species [1] Exudates and resins from some South American plants have been previously reported as sources of potential antimicrobial and antioxidant compounds [2-5]. Argentina and Mexico are the main habitat of resinous plant species belonging to the genera Larrea (Zygophyllaceae). From this genus, four species are widely distributed in Argentina (Larrea ameghinoi, L. cuneifolia, L. divaricata and L. nitida); two of the species also inhabit Chile (L. divaricata and L. nitida) and two species grow in Mexico (L. mexicana and L. tridentata) [6,7]. The species of the genus Larrea in Argentina (Larrea ameghinoi, L. cuneifolia, L. divaricata and L. nitida), vernacular name "jarillas" are used extensively in traditional medicine in Argentina and in Andean communities for the treatment of injuries and bruises, and a good disinfectant of wounds, repellent of insects, for roof construction in rural areas and as a vegetable fuel for cooking food. These uses are also shared with the resinous species Zuccagnia punctata, commonly called "jarilla macho" [5]. Extracts and infusions of aerial parts of most species of this genus have displayed several biological activities such as antimicrobial, antioxidant and inhibitory agents of several enzymes, anti-inflammatory, antitumor and others [8-18]. However, the chemical characterization of the Larrea genus resins, characterized by their high content of phenolics compounds, is still scarce, and their potential antioxidant, antibacterial and nematicidal activities.

In previous reports, several medicinal plants and native fruits growing in Argentina and Chile have been analyzed combining full mass spectra and MSn experiments from quadrupole orbitrap spectrometry (Q-OT-MS), significantly updating the chemical composition and their biological activities, in most of the species investigated. A considerable number of these previous reports have given scientific support to the use or consumption in traditional medicine in both countries [19-25].

The main goals and novelty of this work are the antioxidant, antibacterial and nematicidal effects complemented with the full metabolome polyphenolic profile using a hybrid high-resolution mass spectrometer of the resins from the medicinal plants, L. divaricata and L. nitida, supporting the recognized medicinal properties of this plant as a sustainable way of biomolecules of pharmacological interest.

\section{Materials and Methods}

\subsection{Chemicals}

Ultra-pure water ( $<5 \mu \mathrm{g} / \mathrm{L}$ TOC, (total organic carbon)) was obtained from a water purification system Arium 126 61316-RO, plus an Arium 611 UV unit (Sartorius, Goettingen, Germany). Methanol (HPLC grade) and formic acid (puriss. p.a. for mass spectrometry) from J. T. Baker (Phillipsburg, NJ, USA) were obtained. Dichloromethane (HPLC grade) were from Merck (Santiago, Chile). Commercial Folin-Ciocalteu(FC) reagent, 2,2-diphenyl1-picrylhydrazyl (DPPH), ferric chloride hexahydrate, 2,4,6-tris(2-pyridyl)-s-triazine, trolox, quercetin, gallic acid and DMSO were purchased from Sigma-Aldrich Chem. Co. (St Louis, MO, USA). Nordihydroguaiaretic acid (NDGA), $3^{\prime}$ methyl-nordihydroguaiaretic acid (MNDGA), LnRe and characterized by analysis of their spectroscopic data $\left({ }^{1} \mathrm{H}\right.$ and ${ }^{13} \mathrm{C}$ NMR, MS) were used as standards [26].

\subsection{Plant Material}

Three representative and independent samples of $2 \mathrm{~kg}$ of aerial parts (stems with leaves and flowers) for each species were collected in December 2015, in the Iglesia district, province of San Juan (Argentina) at an altitude of $1800 \mathrm{~m}$ (L. divaricata) and $2700 \mathrm{~m}$ (L. nitida) above sea level. Each sample was collected from ten plants. The two plants belonging to the Zygophyllaceae genera were classified and a voucher specimen was deposited in 
the Laboratory of Natural Products of the University of San Juan (Argentina) under the following voucher number IBT-Ld-1and IBT-Ln-1.

\subsection{Extraction of Yellow-Brown Resin}

L. divaricata Cav (LdRe) and L. nitida Cav (LnRe) resins were obtained by dipping the fresh plant $(100 \mathrm{~g})$ in cold $\mathrm{CH}_{2} \mathrm{Cl}_{2}(\mathrm{DCM}, 1000 \mathrm{~mL})$ at room temperature $\left(30^{\circ} \mathrm{C}\right)$ for $60 \mathrm{~s}$. Each DCM extract was concentrated under pressure at $30^{\circ} \mathrm{C}$ to give a following semisolid yellow-brown residue for each Larrea resin: LdRe resin $12 \mathrm{~g}$, (12\% $w / w$ yield) and LnRe resin $9.5 \mathrm{~g},(9.5 \% w / w$ yield $)$. The extraction procedure was done three times.

\subsection{UHPLC-DAD-MS Instrument and Chromatographic Conditions}

A Thermo Scientific Dionex Ultimate 3000 UHPLC system, hyphenated with a Thermo high resolution Q-Exactive focus mass spectrometer (Thermo, Bremen, Germany) was used for the analysis. The chromatographic system was coupled to the MS with a heated electrospray ionization source II (HESI II). Nitrogen (purity $>99.999 \%$ ) obtained from a Genius NM32LA nitrogen generator (Peak Scientific, Billerica, MA, USA) was employed to produce MS fragmentation. Mass calibration for the Orbitrap spectrometer and HESI parameters were explained in detail and precisely in previous reports $[5,24]$.

Solvent delivery was performed at $1 \mathrm{~mL} / \mathrm{min}$ using ultrapure water supplemented with $1 \%$ formic acid (A) and acetonitrile with $1 \%$ acid formic (B) and a program starting with $5 \% \mathrm{~B}$ at zero time, then maintained $5 \% \mathrm{~B}$ for $5 \mathrm{~min}$, then changing to $30 \% \mathrm{~B}$ within $10 \mathrm{~min}$, then maintaining $30 \% \mathrm{~B}$ for $15 \mathrm{~min}$, then going to $70 \% \mathrm{~B}$ for $5 \mathrm{~min}$, then maintaining $70 \% \mathrm{~B}$ for $10 \mathrm{~min}$, and finally returning to $5 \% \mathrm{~B}$ in $10 \mathrm{~min}$ and keeping this condition for twelve additional minutes to achieve column stabilization before the next injection of $20 \mu \mathrm{L}[5,24]$.

\subsection{Determination of Total Phenolic (TP) and Flavonoid (F) Content}

The total content of phenolic compounds and flavonoids was determined by means of the Folin-Ciocalteu and $\mathrm{AlCl}_{3}$ tests respectively $[5,24]$. The evaluated concentrations of the resins were $1 \mathrm{mg} / \mathrm{mL}$. The results were obtained by using standards (gallic acid and quercetin) and were expressed as equivalent in milligrams to these (mg GAE/g Larrea resin for phenolics and $\mathrm{mg} \mathrm{QE} / \mathrm{g}$ Larrea resin for flavonoids).

\subsection{Antioxidant Activity}

\subsubsection{DPPH Scavenging Activity}

The radical scavenging capacity of both resins was assessed using the DPPH assay, which was explained in detail and precisely for reproducibility in previous reports $[5,24]$. The resins and reference standards were analyzed in the concentration range of 1 and $100 \mu \mathrm{g} / \mathrm{mL}$. The values obtained were expressed as mean $\mathrm{EC}_{50} \pm \mathrm{SD}$ in $\mu \mathrm{g}$ resin $/ \mathrm{mL}$.

\subsubsection{Ferric-Reducing Antioxidant Power Assay (FRAP)}

The reducing power of the Larrea resins was evaluated using the FRAP test, following the protocols already reported explained in detail for correct reproducibility $[5,24]$. Both resins were tested at $1 \mathrm{mg} / \mathrm{mL}$. In addition, a Trolox calibration curve $(0-1 \mathrm{mmol} / \mathrm{L})$ was used. The results obtained were reported as milligrams Trolox equivalent by/grams of resin (mg TE/g resin).

\subsubsection{Trolox Equivalent Antioxidant Activity (TEAC) Assay}

The assay was carried out in the microplate protocol, which has been reported in detailed form for their correct reproducibility in previous reports [5,24]. The LdRe and LnRe resins were dissolved in methanol and mixed with $200 \mu \mathrm{L}$ of ABTS, reading the absorbance at $734 \mathrm{~nm}$ after $4 \mathrm{~min}$. Results from a calibration curve constructed with Trolox (reference compound, $0-1 \mathrm{mmol} / \mathrm{L}$ ) are expressed as equivalent milligrams Trolox by grams resin (mg TE/g resin). 


\subsubsection{Lipid Peroxidation in Human Erythrocytes}

The protocol to lipid peroxidation in human erythrocytes (LP) assay was explained in detail and precisely for reproducibility in previous reports [5,24]. Blood samples were obtained from healthy volunteer donors, and extracted by doctors in Biochemistry belonging to our Research Institute. LdRe and LdRe were assayed 100 and $250 \mu \mathrm{g}$ of Larrea resin/mL, while the reference compound catechin was at $100 \mu \mathrm{g}$ resin $/ \mathrm{mL}$. The results are expressed as a percentage of inhibition of LP in $\mu \mathrm{g}$ of resin $/ \mathrm{mL}$. Healthy volunteer donors gave their informed consent for inclusion before participating in the study. The protocol assay was conducted in accordance with the Declaration of Helsinki, and is included in the approvedproject by CICIT-CA-UNSJ-Argentina (Projectcode 80020190100277SJ, Resolución N 0591-20-R-UNSJ) referred to the ethical and environmental safeguard, and to preserve the hygiene and safety conditions in the activities to be carried out in laboratories.

\subsection{Nematicidal Activity}

The nematicidal effects were determined by the procedure previously described [27]. A pure population of Meloidogyne incognita previously identified was collected from infected Solanum lycopersicum fields from San Juan province, Argentina. Infected roots were washed gently and sterilized with $1 \% \mathrm{NaOCl}$ for $4 \mathrm{~min}$. Under stereoscopic microscope at $1.6 \times \mathrm{egg}$ masses hand-picked. They were incubated in a growth chamber at $27 \pm 1^{\circ} \mathrm{C}$. After hatching, second stage juveniles (J2) were collected up to 3 days old for the assays. For the screening, $50 \mathrm{mg}$ of Larrea resins were dissolved in $98.7 \mathrm{~mL}$ distilled water, $1 \mathrm{~mL} \mathrm{MeOH}$ and $0.3 \mathrm{~mL}$ Tween 20. These solutions were filtered on filter paper and the final concentration was calculated for each one and constituted as the undiluted solution. The final concentrations for LdRe was $0.023 \% w / v$ and for $\operatorname{LnRe}$ was $0.025 \% w / v$, each solution were testing undiluted (1), dilution 1:1 (2) and dilution 1:4 (3) v/v. Thirty J2 were incubated in glass Petri dishes of $50 \mathrm{~mm} \varnothing$ with $10 \mathrm{~mL}$ of Larrea resin and kept in the dark at $28 \pm 1{ }^{\circ} \mathrm{C}$. Regarding the negative control, $10 \mathrm{~mL}$ of purified water was used. At $72 \mathrm{~h}$ under stereoscopic microscope, the vitality of the nematodes was corroborated. Five replicates were made for each treatment and for the negative control. J2 were considered as dead if their bodies were straight with no movement even if physically stimulated with a fine needle. Data were expressed as percentage mortality of $\mathrm{J} 2$ and to calculate the correction $\%$ by natural mortality in control, Schneider-Orelli formula was applied: Corrected $\%=($ mortality $\%$ in treatment-mortality $\%$ in control $)) /((100$-mortality $\%$ in control $)) \times 100$, and they were analyzed by ANOVA and LSD Fisher $\alpha=0.05$ to determine the statistical differences between the means, through InfoStat program (v.2018).

\subsection{Antibacterial Activity}

\subsubsection{Microorganisms}

Gram-positive strains: methicillin-sensitive Staphylococcus aureus ATCC 29213 (MSSA), methicillin-resistant Staphylococcus aureus ATCC 43300 (MRSA), Staphylococcus aureusMQ5097, Streptococcus pyogenes-MQ4 and Streptococcus agalactiae and Gram-negative strains: Escherichia coli ATCC 25922, E.coli-MQ11009, E. coli-MQ11068, E. coli-MQ11062 and E. coliMQ586, from the American Type Culture Collection(ATCC, Rockville, MD, USA) and clinical isolates provided by the Microbiology Laboratory of the Public Dr. Marcial Quiroga, Hospital, San Juan, Argentina (MQ) were used.

\subsubsection{Antibacterial Activity of Larrea Resins}

Minimum inhibitory concentration (MIC) of resins and reference antibiotics Cefotaxime (Argentia ${ }^{\circledR}$, Buenos Aires, Argentina) and Imipenem-cilastatin (Imipecil ${ }^{\circledR}$ ) were carried out by broth microdilution techniques using Mueller Hinton medium, according to Clinical and Laboratory Standards Institute (CLSI) [28]. The LdRe and LnRe resins were tested in triplicate between 0.98 and $250 \mu \mathrm{g} / \mathrm{mL}$. Microorganism suspensions were adjusted in a spectrophotometer with sterile physiological solutions to give a final organism density of the $0.5 \mathrm{McF}$ arland scale $\left(5 \times 10^{5} \mathrm{CFU} / \mathrm{mL}\right)$ A volume of $100 \mu \mathrm{L}$ of inoculum suspension 
was added to each well with the exception of the sterility control. The samples were diluted in DMSO in the assay $(\leq 1 \%)$. The absorbances at $620 \mathrm{~nm}$ were determined in a Multiskan FC Microplate Photometer (Thermo Scientific, Waltham, MA, USA). Minimum inhibitory concentration (MIC in $\mu \mathrm{g} / \mathrm{mL}$ ) was defined as the lowest compounds/extracts concentration showing no visible bacterial growth after incubation time. The minimum bactericidal concentration ( $\mathrm{MBC}$ in $\mu \mathrm{g} / \mathrm{mL}$ ) of Larrea resins and reference compounds was determined as follows: After determining the MIC, an aliquot of $5 \mu \mathrm{L}$ sample was withdrawn from each clear well of the microtiter tray and plated onto a agar plate. Inoculated plates were incubated at $37^{\circ} \mathrm{C}$, and MBCs were recorded after $24 \mathrm{~h}$. The MBC was defined as the lowest concentration of each compound that resulted in total inhibition of visible growth in these plates.

2.8.3. Antibacterial Combinatory Effect between Larrea Resins with Cefotaxime by the Checkerboard Design

The potential antibacterial combinatory effect (synergism) between LdRe, LnRe and reference antibiotic cefotaxime (Cef) was evaluated against methicillin-sensitive Staphylococcus aureus ATCC 25923 (MSSA) and methicillin-resistant S. aureus ATCC 43300 (MRSA), using the checkerboard design [29]. Briefly, LdRe and LnRe were diluted two-fold in the vertical orientation, whereas antibacterial drugs were diluted in the horizontal one. Their respective concentrations were 4,2,1/2,1/4 and 1/8 $\times$ MIC, which were selected based on MIC values previously determined. In each well (microliter plate) was added an inoculum of $5 \times 10^{5}(\mathrm{CFU} / \mathrm{mL})$ and incubated at $37^{\circ} \mathrm{C}(24 \mathrm{~h})$. The checkerboard design are showed in Figure S1 (Supplementary Materials) was as follows:

For each combination, the fractional inhibitory concentration (FIC) was calculated as follows:

$$
\begin{aligned}
F I C_{\text {Larrea resin }} & =M I C_{\text {Larrea in comb. }} / M I C_{\text {Larrea resin alone }} \\
F I C_{C e f} & =M I C_{\text {Cef in comb. }} / M I C_{\text {Cef alone }}
\end{aligned}
$$

Additionally, the fractional inhibitory concentration index (FICI) was determined by following equation:

$$
F I C I=F I C_{\text {Larrea resin }}+F I C_{C e f}
$$

The values obtained were interpreted as follow: synergy effect $(\mathrm{FICI} \leq 0.5)$, no interaction or indifference (FICI > 0.5-4.0), and antagonism (FICI > 4) according to Odds, (2003) [30].

\subsubsection{Dose Reduction Index (DRI)}

The dose reduction index (DRI) determines how manyfolds the dose of an antibiotic in a combination may be reduced at a given effect level compared with the doses of the antibiotic alone. A greater DRI indicates a greater dose reduction for a given effect level [31]. The DRI value for cefotaxime was calculated as follows:

$$
D R I=M I C_{\text {Cef alone }} / M I C_{\text {Cef in comb }}
$$

\subsubsection{Isobolograms}

The potential antibacterial combinatory effect between LdRe and LnRe and cefotaxime antibiotic are showed in normalized Isobolograms, which depicts the results of the checkerboard design [32]. In the Isobolograms, the alone MIC value of Larrea resins (LdRe o LnRe) is represented on the x-axis, and the alone MIC of the cefotaxime on the $y$-axis. The line of no interaction (line of indifference) represents the line connecting these two points. The combinations below this line are a synergistic effect $(\mathrm{FICI} \leq 0.5)$, while combinations above represent an antagonism effect $(\mathrm{FICI}>4)[30]$.

\subsection{Statistical Analysis}

All determinations in total phenolic (TP), flavonoid (FT) and antioxidant assays were made in triplicate using a Multiskan FC Microplate Photometer (Thermo Scien- 
tific, Waltham, MA, USA). The values from triplicates are reported as the mean \pm standard deviation (SD). In addition, the Duncan's test of the InfoStat program (2016 edition, National University of Córdoba, Argentina) was used to determine statistical differences $(p<0.05)$ between the tests used.

\section{Results}

\subsection{UHPLC-OT Analysis of LdRe and LnRe}

The use of full scan mass spectra, using base peaks chromatograms and fragmentation experiments were very useful for the identification of a significant number of flavonoids, epoxylignans and cyclolignans, together with some isomer compounds characteristics of these bioactive plants since the orbital trap provided high-resolution and accurate mass product ion spectra for untargeted analyses within a single run.

Combining full MS spectra and some diagnostic MSn experiments, forty-one compounds were detected in LdRe and LnRe resins, by ultrahigh resolution liquid chromatography Q-orbitrap MS analysis (UHPLC-PDA-Q-OT-MS). From them, the detected and characterized compounds were tentatively proposed as flavonoids, lignans and their derivatives. The generation of molecular formulas was performed using high resolution accurate mass analysis (HRAM) and matching with the isotopic pattern. In this work only negative mode of detection was used. Electrospray negative mode with energy of " 0 " or " 5 " $\mathrm{EV}$ is the most abundantly used method to detect phenolics. Compounds with a phenolics $\mathrm{OH}$ easily lose the proton in electrospray ionization, giving very good and diagnostic parent ions and fragments. Finally, analyses were confirmed using MS/MS data and comparing the fragments found with the literature data. The complete metabolome identification is showed below involving Figures 1-3, Table 1; and Supplementary Material Figure S2.

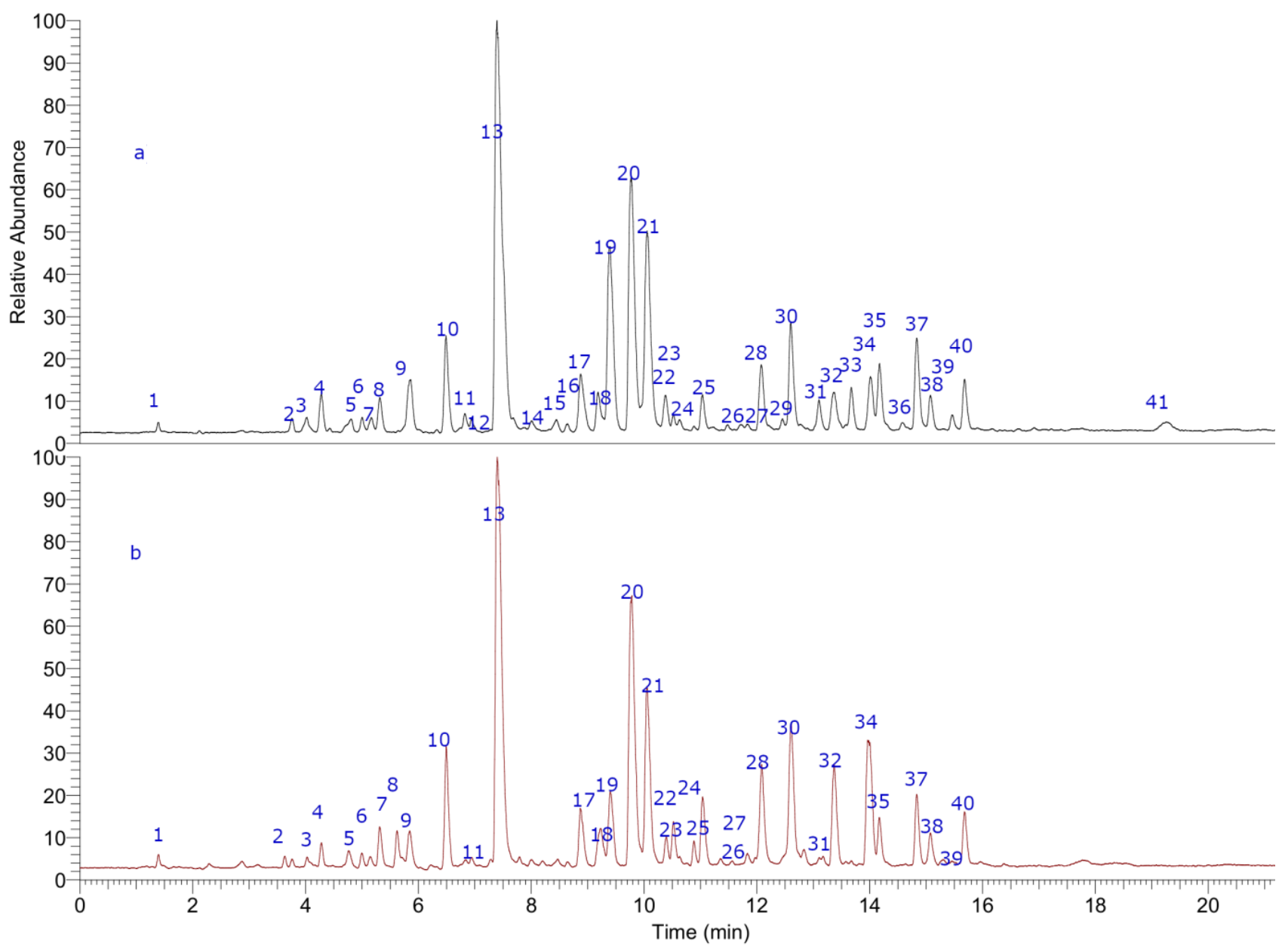

Figure 1. UHPLC-MS (total ion current) chromatograms of LnRe (a) and LdRe (b) resins. 
<smiles>COC(=O)c1cc(OC)cc(O)c1C(=O)c1c(O)cc(C)cc1O</smiles><smiles>COC1=C(O)c2c(oc(-c3ccc(O)c(O)c3)c(OC)c2=O)CC1O</smiles><smiles>COc1ccc(-c2cc(=O)c3c(O)cc(O)cc3o2)cc1O</smiles><smiles>C[C@@H]1[C@@H](c2ccc(O)c(O)c2)O[C@@H](c2ccc(O)c(O)c2)[C@@H]1C</smiles><smiles>COc1cc(-c2oc3cc(O)cc(O)c3c(=O)c2O)ccc1O</smiles><smiles>COc1cc([C@H]2c3cc(O)c(O)cc3CC(C)C2C)ccc1O</smiles><smiles>Cc1c(-c2ccc(O)c(O)c2)oc2cc(O)cc(O)c2c1=O</smiles><smiles>COc1cc([C@H]2c3cc(O)c(O)cc3CC(C)C2C)ccc1O</smiles><smiles>CC1Cc2cc(O)c(O)cc2[C@H](c2ccc(O)cc2)C1C</smiles><smiles>COc1ccc([C@H]2O[C@@H](c3ccc(O)c(O)c3)[C@H](C)[C@H]2C)cc1O</smiles><smiles>CC(=Cc1ccc(O)cc1)C(C)=Cc1ccc(O)c(O)c1</smiles>

Figure 2. Flavonoids, epoxylignans and cyclolignans characterized in LnRe and LdRe resins. 


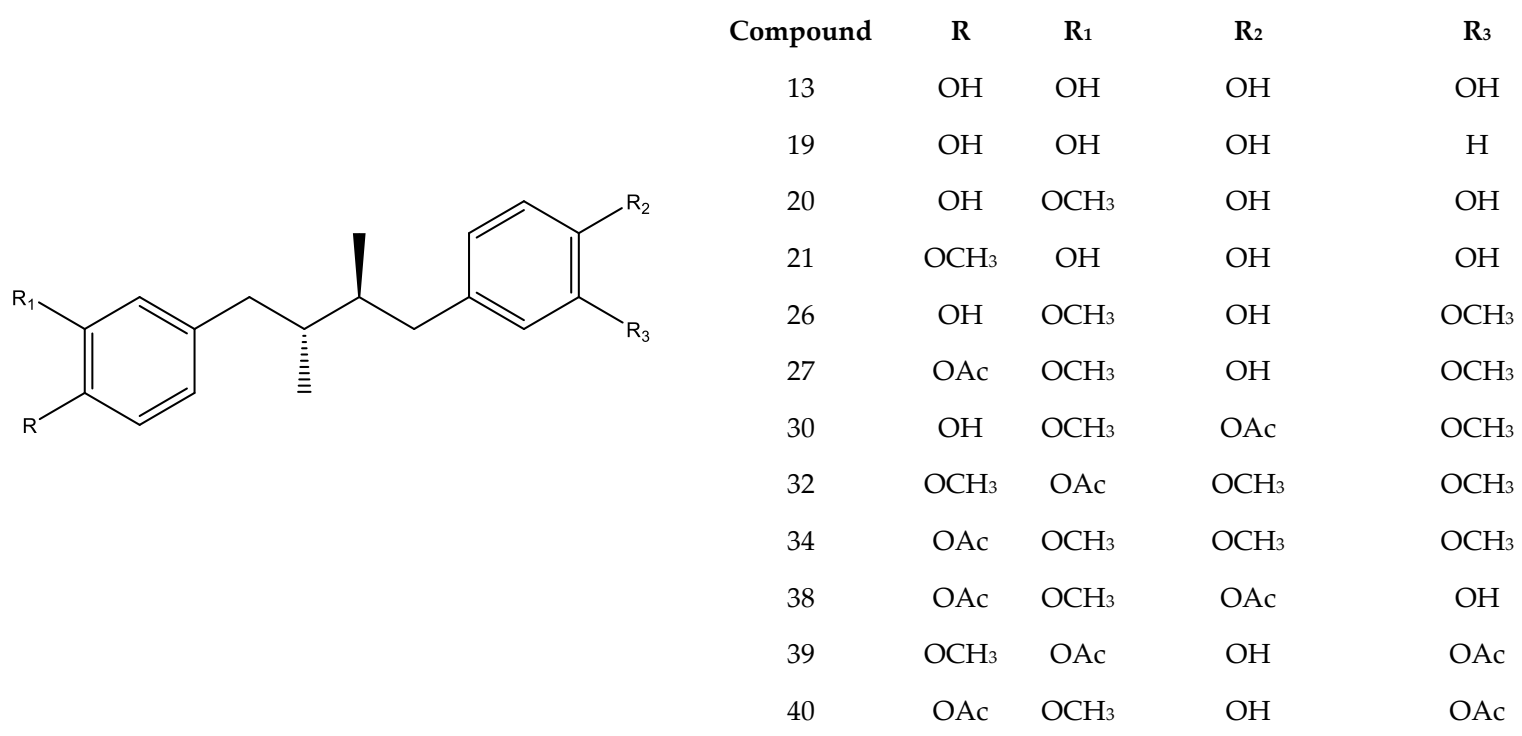

Figure 3. Main NDGA and MNDGA lignans derivatives characterized in LnRe and LdRe resins.

Table 1. Ultrahigh resolution liquid chromatography orbitrap MS analysis (UHPLC-PDA-MS) orbitrap mass spectral data of LdRe and LnRe resins.

\begin{tabular}{|c|c|c|c|c|c|c|c|c|}
\hline Peak & $\begin{array}{c}\mathrm{T}_{\mathrm{R}} \\
\text { (min.) }\end{array}$ & $\begin{array}{c}\text { Tentative } \\
\text { Identification }\end{array}$ & {$[\mathbf{M}-\mathbf{H}]^{-}$} & $\begin{array}{c}\text { UV Max } \\
\text { nm }\end{array}$ & $\begin{array}{l}\text { Theoretical } \\
\text { Mass }(\mathrm{m} / \mathrm{z})\end{array}$ & $\begin{array}{l}\text { Measured } \\
\text { Mass }(m / z)\end{array}$ & 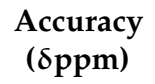 & 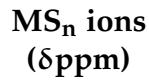 \\
\hline 1 & 1.40 & Isosulochrin & $\mathrm{C}_{17} \mathrm{H}_{15} \mathrm{O}_{7}^{-}$ & $278-337$ & 331.08123 & 331.08212 & 2.69 & 146.96791 \\
\hline 2 & 3.65 & Axillarin & $\mathrm{C}_{17} \mathrm{H}_{13} \mathrm{O}_{8}$ & $2890-340$ & 345.06049 & 345.06143 & 1.28 & 146.96791 \\
\hline 3 & 4.10 & $\begin{array}{l}\text { Diosmetin } \\
\text { meso-(rel }\end{array}$ & $\mathrm{C}_{16} \mathrm{H}_{11} \mathrm{O}_{6}^{-}$ & $270-344$ & 299.05501 & 299.05597 & 3.19 & $\begin{array}{l}271.06097 \\
267.06481\end{array}$ \\
\hline 4 & $\begin{array}{l}4.26- \\
4.30\end{array}$ & $\begin{array}{c}\left.7 \mathrm{~S}, 8 \mathrm{~S}, 7^{\prime} \mathrm{R}, 8^{\prime} \mathrm{R}\right)- \\
3,4,3^{\prime}, 4^{\prime}- \\
\text { tetrahydroxy- } \\
7,7^{\prime} \text {-epoxylignan }\end{array}$ & $\mathrm{C}_{18} \mathrm{H}_{19} \mathrm{O}_{5}^{-}$ & $233-284$ & 315.12380 & 315.12381 & & $\begin{array}{l}187.07582 ; \\
165.05512 ; \\
137.02363 ; \\
109.02862\end{array}$ \\
\hline 5 & 4.80 & $\begin{array}{c}\text { Isorhamnetin } \\
3,4,3^{\prime}, 4^{\prime}-\end{array}$ & $\mathrm{C}_{16} \mathrm{H}_{11} \mathrm{O}_{7}^{-}$ & & 315.04993 & 315.05084 & 2.89 & 299.09216 \\
\hline 6 & 5.00 & $\begin{array}{c}\text { tetrahydroxy } \\
6,7^{\prime}-\text { Cyclolignan }\end{array}$ & $\mathrm{C}_{18} \mathrm{H}_{19} \mathrm{O}_{4}^{-}$ & $233-284$ & 299.12888 & 299.12872 & 3.13 & $\begin{array}{l}\text { 241.05080; } \\
190.92799\end{array}$ \\
\hline 7 & 5.33 & 3 Methylluteolin & $\mathrm{C}_{16} \mathrm{H}_{11} \mathrm{O}_{6}{ }^{-}$ & $275-330$ & 299.05501 & 299.05597 & 3.19 & 271.06097 \\
\hline 8 & 5.65 & Rhamnetin & $\mathrm{C}_{16} \mathrm{H}_{11} \mathrm{O}_{7}^{-}$ & $256-367$ & 315.04993 & 315.05099 & 3.38 & 299.09216 \\
\hline 9 & 5.80 & $\begin{array}{l}\text { Tricin } \\
3,4,3^{\prime}, 4^{\prime}-\end{array}$ & $\mathrm{C}_{17} \mathrm{H}_{13} \mathrm{O}_{7}^{-}$ & $256-367$ & 329.06558 & 329.06644 & 2.60 & $\begin{array}{c}285.0401 \\
243.06482\end{array}$ \\
\hline 10 & 6.50 & $\begin{array}{c}\text { tetrahydroxy } \\
6,7^{\prime} \text {-cyclolignan } \\
\text { isomer }\end{array}$ & $\mathrm{C}_{18} \mathrm{H}_{19} \mathrm{O}_{4}^{-}$ & $233-284$ & 299.12888 & 299.12872 & 3.13 & $\begin{array}{c}189.09164 ; \\
149.0600 ; \\
123.0443\end{array}$ \\
\hline 11 & 6.80 & NDGA isomer & $\mathrm{C}_{18} \mathrm{H}_{19} \mathrm{O}_{4}^{-}$ & $228-283$ & 301.14344 & 301.14435 & 3.03 & $\begin{array}{l}174.95743 \\
122.03708\end{array}$ \\
\hline 12 & 6.90 & $\begin{array}{l}\text { Norisoguaiacin } \\
\text { isomer }\end{array}$ & $\mathrm{C}_{19} \mathrm{H}_{21} \mathrm{O}_{4}^{-}$ & $231-286$ & 313.14453 & 313.14432 & 2.81 & 247.09711 \\
\hline 13 & 7.40 & NDGA & $\mathrm{C}_{18} \mathrm{H}_{21} \mathrm{O}_{4}^{-}$ & $228-283$ & 301.14344 & 301.14429 & 3.01 & $\begin{array}{l}174.95743 ; \\
154.02631 ; \\
137.02362 ; \\
122.03708\end{array}$ \\
\hline 14 & 7.94 & NDGA isomer & $\mathrm{C}_{18} \mathrm{H}_{21} \mathrm{O}_{4}^{-}$ & $228-283$ & 301.14344 & 301.14471 & 3.06 & $\begin{array}{l}243.06614 \\
122.0367\end{array}$ \\
\hline 15 & 8.42 & Kaempferide & $\mathrm{C}_{16} \mathrm{H}_{11} \mathrm{O}_{6}^{-}$ & 269-358 & 299.05501 & 299.05594 & 3.09 & $\begin{array}{c}285.04053 \\
255.0279\end{array}$ \\
\hline
\end{tabular}


Table 1. Cont.

\begin{tabular}{|c|c|c|c|c|c|c|c|c|}
\hline Peak & $\begin{array}{c}\mathrm{T}_{\mathrm{R}} \\
\text { (min.) }\end{array}$ & $\begin{array}{c}\text { Tentative } \\
\text { Identification }\end{array}$ & {$[\mathbf{M}-\mathbf{H}]^{-}$} & $\begin{array}{l}\text { UV Max } \\
\text { nm }\end{array}$ & $\begin{array}{l}\text { Theoretical } \\
\text { Mass }(m / z)\end{array}$ & $\begin{array}{l}\text { Measured } \\
\text { Mass }(m / z)\end{array}$ & $\begin{array}{l}\text { Accuracy } \\
(\delta p p m)\end{array}$ & $\begin{array}{l}\mathrm{MS}_{\mathrm{n}} \text { ions } \\
(\delta \mathrm{ppm})\end{array}$ \\
\hline 17 & 8.85 & $\begin{array}{l}\text { Trihydroxy- } \\
\text { 6,7'cyclolignan }\end{array}$ & $\mathrm{C}_{19} \mathrm{H}_{18} \mathrm{O}_{3}^{-}$ & 233-284 & 283.13397 & 283.06111 & & $\begin{array}{l}\text { 268.03041; } \\
\text { 240.04212; } \\
211.03951 ; \\
117.03852\end{array}$ \\
\hline 18 & 9.15 & $\begin{array}{c}\left(7 S, 8 S, 7^{\prime} R, 8^{\prime} R\right)- \\
3,3^{\prime}, 4^{\prime}- \\
\text { trihydroxy-4- } \\
\text { methoxy-7,7'- } \\
\text { epoxylignan }\end{array}$ & $\mathrm{C}_{19} \mathrm{H}_{21} \mathrm{O}_{5}^{-}$ & $230-283$ & 329.13945 & 329.17551 & 2.34 & $\begin{array}{l}301.14410 \\
263.12848\end{array}$ \\
\hline 19 & 9.37 & $\begin{array}{l}\text { 4-[4-(4- hydroxy- } \\
\text { phenyl)-2,3- } \\
\text { dimethyl-butyl]- } \\
\text { benzene-1,2-diol }\end{array}$ & $\mathrm{C}_{18} \mathrm{H}_{21} \mathrm{O}_{3}^{-}$ & 282 & 285.14852 & 285.14948 & 3.0 & $\begin{array}{l}154.02643 ; \\
137.02362 ; \\
122.03652\end{array}$ \\
\hline 20 & 9.75 & $3^{\prime}$-MNDGA & $\mathrm{C}_{19} \mathrm{H}_{23} \mathrm{O}_{4}^{-}$ & $230-283$ & 315.15909 & 315. 15981 & 1.99 & $\begin{array}{l}\text { 300.13622; } \\
\text { 285.11272; } \\
\text { 177.09142; } \\
\text { 149.06005; } \\
122.03653 .\end{array}$ \\
\hline 21 & 10.06 & $4^{\prime}-\mathrm{MNDGA}$ & $\mathrm{C}_{19} \mathrm{H}_{23} \mathrm{O}_{4}^{-}$ & $230-283$ & 315.15909 & 315.15991 & 1.62 & $\begin{array}{l}300.13626 ; \\
285.11282 ; \\
177.09142 ; \\
149.06001 ; \\
122.03653\end{array}$ \\
\hline 22 & 10.36 & \multicolumn{2}{|c|}{$\begin{array}{l}\text { Lavandulilkaempfer }{ }_{25} \mathrm{H}_{25} \mathrm{O}_{6}{ }^{-} \\
\text {Unknown }\end{array}$} & 272 & 421.16456 & 421.16541 & 1.99 & 301.144134 \\
\hline 23 & 11.04 & $\begin{array}{c}\text { NDGA } \\
\text { derivative } \\
\text { Unknown }\end{array}$ & $\mathrm{C}_{27} \mathrm{H}_{29} \mathrm{O}_{6}^{-}$ & 280 & 449.19587 & 449.19659 & 1.62 & 301.144261 \\
\hline 24 & 11.48 & $\begin{array}{l}\text { NDGA } \\
\text { derivative } \\
\text { Unknown }\end{array}$ & $\mathrm{C}_{24} \mathrm{H}_{31} \mathrm{O}_{6}^{-}$ & 280 & 415.21152 & 415.21222 & 1.69 & 301.14425; \\
\hline 25 & 11.71 & $\begin{array}{c}\text { NDGA } \\
\text { derivative }\end{array}$ & $\mathrm{C}_{27} \mathrm{H}_{27} \mathrm{O}_{6}^{-}$ & 280 & 447.18022 & 447.18079 & 1.27 & 301.14423 \\
\hline 26 & 11.81 & $\begin{array}{l}\text { Dihydroguaiaretic } \\
\text { acid }\end{array}$ & $\mathrm{C}_{20} \mathrm{H}_{25} \mathrm{O}_{4}^{-}$ & $230-283$ & 329.17474 & 329.17557 & 2.52 & $\begin{array}{l}301.14410 ; \\
263.12848\end{array}$ \\
\hline 27 & 12.07 & $\begin{array}{l}\text { Dihydroguaiaretic } \\
\text { acid } 4^{\prime} \text {-O-acetate }\end{array}$ & $\mathrm{C}_{22} \mathrm{H}_{27} \mathrm{O}_{5}^{-}$ & 283 & 371.18530 & 371.18597 & 1.81 & 301.14420 \\
\hline 28 & 12.4 & $\begin{array}{c}\text { Reduced NDGA } \\
\text { derivative }\end{array}$ & $\mathrm{C}_{18} \mathrm{H}_{17} \mathrm{O}_{3}^{-}$ & 283 & 281.11722 & 281.11813 & 3.24 & 301.14420 \\
\hline 29 & 12.85 & $\begin{array}{l}\text { Unknown } \\
\text { NDGA } \\
\text { derivative }\end{array}$ & $\mathrm{C}_{25} \mathrm{H}_{25} \mathrm{O}_{5}^{-}$ & 283 & 405.16965 & 405.17147 & 2.02 & 301.14420 \\
\hline 30 & 12.61 & $\begin{array}{l}\text { Dihydroguaiaretic } \\
\text { acid } 4 " \text {-O-acetate }\end{array}$ & $\mathrm{C}_{22} \mathrm{H}_{27} \mathrm{O}_{5}^{-}$ & $276-352$ & 371.18530 & 371.18595 & 1.73 & 301.14420 \\
\hline 31 & 13.11 & NDGA isomer & $\mathrm{C}_{18} \mathrm{H}_{21} \mathrm{O}_{4}^{-}$ & 283 & 301.14344 & 301.14429 & 2.82 & $\begin{array}{l}\text { 174.95712; } \\
122.03708\end{array}$ \\
\hline 32 & 13.37 & $\begin{array}{c}3^{\prime \prime}, 4^{\prime \prime}, 4^{\prime}- \\
\text { Trymethyl } \\
\text { NDGA } 3^{\prime} \text {-acetate }\end{array}$ & $\mathrm{C}_{23} \mathrm{H}_{29} \mathrm{O}_{5}^{-}$ & 277 & 385.20195 & 385.20175 & 2.02 & 301.14423 \\
\hline 33 & 13.68 & NDGA isomer & $\mathrm{C}_{18} \mathrm{H}_{21} \mathrm{O}_{4}^{-}$ & 280 & 301.14344 & 301.14429 & 2.82 & $\begin{array}{l}\text { 174.95743; } \\
122.03708\end{array}$ \\
\hline 34 & 14.00 & $\begin{array}{c}3^{\prime \prime}, 4^{\prime \prime}, 3^{\prime}- \\
\text { Trymethyl } \\
\text { NDGA } 4^{\prime} \text {-acetate }\end{array}$ & $\mathrm{C}_{23} \mathrm{H}_{29} \mathrm{O}_{5}^{-}$ & 283 & 385.20195 & 385.20172 & 2.00 & $\begin{array}{l}301.14426 ; \\
122.03667\end{array}$ \\
\hline 35 & 14.19 & $\begin{array}{c}\text { Unknown } \\
\text { NDGA } \\
\text { derivative }\end{array}$ & $\mathrm{C}_{27} \mathrm{H}_{29} \mathrm{O}_{5}^{-}$ & 283 & 433.20095 & 433.20142 & 1.07 & 301.14429 \\
\hline
\end{tabular}


Table 1. Cont.

\begin{tabular}{|c|c|c|c|c|c|c|c|c|}
\hline Peak & $\begin{array}{c}T_{R} \\
\text { (min.) }\end{array}$ & $\begin{array}{c}\text { Tentative } \\
\text { Identification }\end{array}$ & {$[\mathbf{M}-\mathbf{H}]^{-}$} & $\begin{array}{c}\text { UV Max } \\
\text { nm }\end{array}$ & $\begin{array}{l}\text { Theoretical } \\
\text { Mass }(m / z)\end{array}$ & $\begin{array}{l}\text { Measured } \\
\text { Mass }(m / z)\end{array}$ & 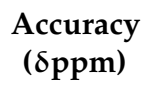 & $\begin{array}{c}\text { MS }_{\mathrm{n}} \text { ions } \\
(\delta \mathrm{ppm})\end{array}$ \\
\hline 36 & 14.56 & $\begin{array}{c}\text { Unknown } \\
\text { NDGA } \\
\text { derivative }\end{array}$ & $\mathrm{C}_{26} \mathrm{H}_{33} \mathrm{O}_{6}^{-}$ & 283 & 441.22717 & 441.22781 & 1.46 & 301.14429 \\
\hline 37 & 14.83 & $\begin{array}{l}\text { Unknown } \\
\text { NDGA } \\
\text { derivative }\end{array}$ & $\mathrm{C}_{27} \mathrm{H}_{29} \mathrm{O}_{5}^{-}$ & 283 & 433.20095 & 433.20157 & 1.07 & 301.14426 \\
\hline 38 & 15.08 & $\begin{array}{c}3^{\prime} \text { MNDGA 3", } \\
4^{\prime} \text {-diacetate }\end{array}$ & $\mathrm{C}_{24} \mathrm{H}_{31} \mathrm{O}_{5}^{-}$ & 283 & 399.21660 & 399.21738 & 1.94 & 301.14426 \\
\hline 39 & 15.45 & $\begin{array}{l}4^{\prime} \mathrm{MNDGA} 3^{\prime} \\
4^{\prime \prime} \text {-diacetate }\end{array}$ & $\mathrm{C}_{24} \mathrm{H}_{31} \mathrm{O}_{5}^{-}$ & 283 & 399.21660 & 399.21729 & 1.71 & 301.14426 \\
\hline 40 & 15.68 & $\begin{array}{l}3^{\prime} \text { MNDGA } 4^{\prime \prime}, \\
4^{\prime} \text {-diacetate }\end{array}$ & $\mathrm{C}_{24} \mathrm{H}_{31} \mathrm{O}_{5}^{-}$ & 283 & 399.21660 & 399.21738 & 1.94 & 301.14429 \\
\hline 41 & 19.19 & Unknown & $\mathrm{C}_{24} \mathrm{H}_{23} \mathrm{O}_{3}^{-}$ & - & 359.16427 & 359.16513 & 2.67 & 235.92734 \\
\hline
\end{tabular}

Forty-one phenolics compounds were detected and tentatively identified by ultrahigh resolution liquid chromatography orbitrap MS analysis in LdRe and LnRe resins. Of these, one was a phenolic compound (1), eight wereflavonoids $(2,3,5,7,8,9,15$ and 22) and twenty-nine tentatively proposed as lignans $(4,6,10,11,12,13,16,17,18,19,20$, $21,23,24,25,26,27,28,29,30,31,32,33,34,35,36,37,38,39$ and 40). From them, a significant number were assigned as NDGA and its derivatives, supported by molecular mass, fragmentation patterns, bibliographic references and databases as SciFinder, MassBank of North America (MoNA) and UHPLC-MS internal library. Finally, peak 41 was assigned as unknown. Peak 4, 13, 18, 19 and 20 were identified as meso-(rel $\left.7 \mathrm{~S}, 8 \mathrm{~S}, 7^{\prime} \mathrm{R}, 8^{\prime} \mathrm{R}\right)-3,4,3^{\prime}, 4^{\prime}$-tetrahydroxy-7, $7^{\prime}$-epoxylignan; nordihydroguaiaretic acid (NDGA); $\left(7 \mathrm{~S}, 8 \mathrm{~S}, 7^{\prime} \mathrm{R}, 8^{\prime} \mathrm{R}\right)$ - 3,3', $4^{\prime}$-trihydroxy-4-methoxy-7, $7^{\prime}$-epoxylignan; (4-[4-(4-hydroxy-phenyl)2,3-dimethyl-butyl]-benzene-1,2-diol) and $3^{\prime}$ methyl-nordihydroguaiaretic acid (MNDGA) respectively by spiking experiments with available standards previously isolated [26]. In a similar way peak 21 was proposed as a $4^{\prime}$-MNDGA, an isomer of 20, supported by their identically measured accurate mass and major diagnostic MS ions [26].

The compounds 11, 14, 31 and $\mathbf{3 3}$ were tentatively identified as isomers of NDGA (13), from them, in the compounds 31 and 33; the parent ion (301.14429) produced the same major diagnostic MS ions at $\mathrm{m} / \mathrm{z} 174.95743$ and $\mathrm{m} / \mathrm{z} 122.03708$ confirming these proposed isomers. A synthetic route to the naturally occurring nordihydroguaiaretic acid and its non-meso isomer starting from the commercially available (3,4-dimethoxyphenyl) acetone has been reported [33]. On the other hand, peaks 26 with a at $\mathrm{m} / \mathrm{z} 329.17551$ was tentatively identified as meso-dihydroguaiaretic acid [34,35]; while $[\mathrm{M}-\mathrm{H}]^{-}$ion peak 28 with a $[\mathrm{M}-\mathrm{H}]^{-}$ion at $m / z 281.11813$ was tentatively identified as reduced NDGA derivative (Supplementary material).

Peaks 6 and 10 with a $[\mathrm{M}-\mathrm{H}]^{-}$ion at $m / z 299.12872$ were proposed as 3,4,3', $4^{\prime}-$ tetrahydroxy $6,7^{\prime}$-cyclolignan isomers. Peak 12 and 16 with a $[\mathrm{M}-\mathrm{H}]^{-}$ion at $m / z 313.14432$ were assigned to norisoguaicin and its isomer, both compounds and other lignans have been previously isolated from L. divaricata, L. nitida and L. tridentate [34-39].

Peak 17 was proposed as trihydroxy-6,7' cyclolignan with a $[\mathrm{M}-\mathrm{H}]^{-}$ion at $\mathrm{m} / \mathrm{z}$ 283.0611 [40]. Fourteen peaks including 23, 24, 25, 27, 29, 30, $\mathbf{3 2}$ and 34-40 were proposed as NDGA derivatives, showing all of them as major diagnostic MS ions between 301.14420 and 301.14429 Daltons. From them, peaks 27 and 30, both with a $[\mathrm{M}-\mathrm{H}]^{-}$ion at $m / z 371.18595$ were tentatively identified as dihydroguaiaretic acid acetates, $\left(4^{\prime}-\mathrm{O}\right.$ acetate and $4-\mathrm{O}$ acetate respectively), which were isolated previously from $L$ tridentata extracts [38,40,41]. Peaks 32 and 34 were tentatively proposed as the tri-methoxylated and acetylated isomers: $3^{\prime \prime}, 4^{\prime \prime}, 4^{\prime}$ trymethyl NDGA3'-acetate and $3^{\prime \prime}, 4^{\prime \prime}, 3^{\prime}$-trymethyl NDGA4'-acetate, with $[\mathrm{M}-\mathrm{H}]^{-}$ions at $m / z 385.20175$ and 385.20172 respectively, this is in concordance with tri-methoxylated NDGA derivatives previously isolated from the same source $[38,40,41]$. Peaks $\mathbf{3 8}$ and 
40 producing the same $[\mathrm{M}-\mathrm{H}]^{-}$ion at $m / z 399.21738$ and peak 39 with a $[\mathrm{M}-\mathrm{H}]^{-}$ion at $m / z 399.21729$ yielded the same mayor diagnostic MS ion at $\mathrm{m} / \mathrm{z} 301.14429$, thorough losses of the substituent's and thus the three compounds were proposed as MNDGA diacetate derivatives (3'Methyl NDGA 3", 4'-diacetate, 4'Methyl NDGA 3', 4"-diacetate and $3^{\prime}$ Methyl NDGA 4", 4'-diacetate, respectively). According to our knowledge, the isolation and identification of phenolics, flavonoids and lignans from extracts of $L$. divaricata and L. nitida have been reported in some previous works. From L. nitida collected in Chile, norisoguaicin16, nordihydroguaiaretic acid (13, NDGA) and ferulic acid have previously been reported [36]. Additionally, the antifungal bioassay-guided isolation of L. nitida resin ( $\mathrm{LnRe}$ ) led to the isolation of 4, 13, 16, 18, 20 and 21 [26]. Recently, from an ethanol-water extract of the species L. divaricata, L. nitida and L. cuneifolia, through a study by HPLCMS in the negative mode, twelve phenolics compounds were characterized, including, quercetin-hexoside, quercetin-methyl ether hexoside and lignans $(4,6,13,17,18,19,20$ and 21) [39]. On the other hand, previous phytochemical investigation of the phenolics from L. divaricata led to the isolation and characterization of the lignans nor-dihydroguaiaretic acid (13), dihydroguaiaretic acid (26), norisoguaiacin (16), $3^{\prime}$-demethoxyisoguaiacin and several flavonoids, including kaempferol 3-methyl ether (isokaempferide), isorhamnetin (3) and luteolin $3^{\prime}$-methyl ether [42]. In this report, forty-one compounds were detected in LnRe resin by ultrahigh resolution liquid chromatography orbitrap MS analysis (UHPLCPDA-OT-MS). From them, as far as we know, one phenolic compound (1), eight flavonoids $(2,3,5,7,8,9,15$ and 22), and nineteen lignans $(14,23,24,25,26,27,28,29,30,31,32,33,34$, $35,36,37,38,39$ and 40 ) were reported here for the first time in these species, significantly updating the chemical composition of these interesting native plants.

\subsection{Total Phenolics and Flavonoids Content; Antioxidant, Nematicidal and Antibacterial Activities}

The yellow-brown resins of the two Larrea species showed a strong free radical scavenging in the DPPH assay, with EC50 values around $8.4 \mu \mathrm{g}$ resin $/ \mathrm{mL}$ (Table 2). These values were comparable to the activity shown by recognized antioxidant compounds such as catechin or quercetin. In the same way, the yellow-brown resins exhibited a strong effect in FRAP and TEAC trials. On the other hand, yellow-brown resins of the two Larrea presented a high content of TP, highlighting LnRe collected in the Andean mountains over $2500 \mathrm{~m}$ above sea level with a value of $459 \mathrm{mg}$ GAE/g LnRe, of which approximately 8.8 percent correspond to flavonoids ( $40.8 \mathrm{mg} \mathrm{QE} / \mathrm{g}$ LnRe) (Table 2). Additionally, both resins exhibited a strong inhibition of lipoperoxidation with LP values between 81 and $82 \%$ at $100 \mu \mathrm{g}$ resin $/ \mathrm{mL}$ compared to catechin, which showed $70 \%$ of LP at the same concentration [24]. Other Andean medicinal plants collected in the Andean region of the province of San Juan, located in the central west of Argentina, also showed strong antioxidant properties and high contents of phenolics compounds, which were characterized mainly by flavonoids, chalcones and phenolics of diverse structures [3,5,21].

Table 2. Antioxidant properties and total phenolics and flavonoids content of LnRe and LdRe.

\begin{tabular}{ccc}
\hline Assay & LnRe & LdRe \\
\hline Content of phenols & & \\
Total phenolics(mg GAE/g resin) & $459.9 \pm 10.07$ & $390.46 \pm 6.08$ \\
Flavonoids (mg QE/g resin) & $40.8 \pm 0.8$ & $24.7 \pm 1.8$ \\
Antioxidant & & \\
DPPH (EC 5 in $\mu \mathrm{g}$ resin/mL) & $8.41 \pm 0.04$ & $8.42 \pm 0.44$ \\
FRAP(mgTE/g resin) & $1.94 \pm 0.20$ & $1.86 \pm 0.16$ \\
TEAC (mgTE/g resin) & $1.08 \pm 0.07$ & $1.09 \pm 0.06$ \\
Percentage LP (at 100 $\mu \mathrm{g}$ resin/mL) & $81.97 \pm 0.11$ & $81.03 \pm 0.01$ \\
\hline
\end{tabular}

No significant differences were found between the three samples. ANOVA (analysis of variance) followed by Dunnett's comparison test was used (significance $p<0.05$ ). 
Meloidogyne spp., which causes root-knot diseases in plants, is the most economically important plant-parasitic nematodes worldwide. Due to the harmful effects on humans and the environment, the chemical compounds that have been used, over decades, for the effective control of nematodes, have been losing application fields and opening the way to the need for new nematicides, friendly to the environment. Biopesticides and specifically bionematicides of botanical origin constitute a desirable component of pest management technology and practices [43]. The nematicidal activity of LnRe and LdRe against J2 M. incognita is shown in Table 3. Both resins displayed a less nematicidal activity at $72 \mathrm{~h}$ to three concentrations assayed.

Table 3. Nematicidal activity of LnRe and LdRe resins againstJ2 M. incognita.

\begin{tabular}{ccccc}
\hline \multicolumn{5}{c}{ \% Mortality Corrected } \\
\hline Resins & Concentration & & \\
\hline & & $24 \mathrm{~h}$ & $48 \mathrm{~h}$ & $72 \mathrm{~h}$ \\
\hline \multirow{2}{*}{ LnRe } & 1 & $3.49 \pm 3.24 \mathrm{a}$ & $4.77 \pm 3.89 \mathrm{a}$ & $10.44 \pm 1.37 \mathrm{ab}$ \\
& 2 & $5.52 \pm 3.54 \mathrm{a}$ & $11.77 \pm 3.27 \mathrm{ab}$ & $17.76 \pm 2.27 \mathrm{bc}$ \\
& 3 & $6.47 \pm 4.42 \mathrm{ab}$ & $11.13 \pm 3.15 \mathrm{ab}$ & $8.67 \pm 2.73 \mathrm{a}$ \\
\hline \multirow{2}{*}{ LdRe } & 1 & $9.81 \pm 1.45 \mathrm{ab}$ & $16.23 \pm 1.86 \mathrm{bcd}$ & $24.03 \pm 1.53 \mathrm{cde}$ \\
& 2 & $3.95 \pm 3.83 \mathrm{a}$ & $13.98 \pm 1.65 \mathrm{bc}$ & $28.56 \pm 2.27 \mathrm{cde}$ \\
& 3 & $5.91 \pm 4.69 \mathrm{a}$ & $15.61 \pm 2.91 \mathrm{bc}$ & $20.76 \pm 1.64 \mathrm{~cd}$ \\
\hline
\end{tabular}

Mean percent between rows at each column with different letters are significantly different $(p=0.05)$ according to LSD Fisher.

Regarding the antibacterial activity, LdRe and LdRe showed a very strong activity against MSSA and MRSAwith a MIC values $=16 \mu \mathrm{g} / \mathrm{mL}$ (Table 4). The antimicrobial activity of medicinal plants is considered very interesting with MICs values $<100 \mu \mathrm{g} / \mathrm{mL}$ for extracts and MIC values $<10 \mu \mathrm{g} / \mathrm{mL}$ for isolated compounds [44]. Additionally, LdRe showed activity against Streptococcus pyogenes-MQ4and LnRe was active against Streptococcus agalactiae (MIC $=62.5 \mu \mathrm{g} / \mathrm{mL}$ ). In respect to Gram (-) bacteria, E. coli-MQ586 was more sensitive to both resins (MIC $=62.5 \mu \mathrm{g} / \mathrm{mL}$ ), while these displayed a moderated antibacterial activity against E. coli ATCC 25922, E. coli MQ-11009, E. coli MQ 11068 and E. coli MQ-11062 (MICs values between 250 and $500 \mu \mathrm{g} / \mathrm{mL}$ ). In a previous report, 3'methyl-nordihydroguaiaretic acid (MNDGA)20, nordihydroguaiaretic acid (NDGA) 13 and a NDGA derivative 19, displayed strong activity against Trichophyton mentagrophytes, T. rubrum and Microsporum gypseum (MICs between 15.6 and $31.25 \mu \mathrm{g} / \mathrm{mL}$ ). In the same study, the lignans $\mathbf{2 0}$ and $\mathbf{1 3}$ showed activities against clinical isolates of Candidas spp., Cryptococcus spp., T. rubrum and T. mentagrophytes (MICs and MFCs between 31.25 and $62.5 \mu \mathrm{g} / \mathrm{mL}$ ).

On the other hand, the combined use of plant extracts with commercial antibiotics is an alternative against microbial infections $[45,46]$. The potential of Larrea resins in combination with cefotaxime for the development of a new antimicrobial source useful for the treatment of infections associated to MSSA and MRSA, was evaluated using the checkerboard design (Figure S1). The results are showed in Table 5 and Figure 4. The combination of LdRe or LnRe with reference antibiotic cefotaxime showed an indifferent effect with FICI values $=1-1.25$ (Equations (1) and (2). In addition, a moderated decrease of the individual MIC value of the commercial drug cefotaxime was observed (DRI $=2-4)$, as indicative of a greater adjuvant potential capacity for a given effect level, which may be due mainly to the polyphenols present in the Larrea resins $[47,48]$. This justifies the search for alternative therapies as the combination of commercial drugs and natural products. 
Table 4. Antibacterial activity of LdRe and LnRe resins (minimum inhibitory concentrations (MICs) and minimum bactericidal concentrations (MBCs) in $\mu \mathrm{g}$ resin $/ \mathrm{mL}$ ).

\begin{tabular}{|c|c|c|c|c|c|c|c|c|}
\hline \multirow{3}{*}{$\begin{array}{l}\text { Bacterias } \\
\text { Gram (+) } \\
\end{array}$} & \multicolumn{4}{|c|}{ Larrea Resins } & \multicolumn{4}{|c|}{ Reference Antibiotics } \\
\hline & \multicolumn{2}{|c|}{ LnRe } & \multicolumn{2}{|c|}{ LdRe } & \multicolumn{2}{|c|}{ Cefotaxime } & \multicolumn{2}{|c|}{ Imipecil } \\
\hline & MIC & $\mathrm{MBC}$ & MIC & $\mathrm{MBC}$ & MIC & $\mathrm{MBC}$ & MIC & $\mathrm{MBC}$ \\
\hline MSSA & 16 & 32.5 & 16 & 32.5 & 0.5 & 0.5 & 0.5 & 0.5 \\
\hline MRSA & 16 & 32.5 & 16 & 16 & 0.5 & 0.5 & 0.5 & 0.5 \\
\hline Staphylococcus aureus-MQ5097 & 250 & 250 & 250 & 250 & 0.8 & 1 & 1 & 1 \\
\hline Streptococcuspyogenes-MQ4 & 250 & 250 & 62.5 & 62.5 & 0.25 & 0.5 & 0,5 & 0.5 \\
\hline Streptococcus agalactiae & 62.5 & 62.5 & 125 & 125 & 0.5 & 0.5 & 0,5 & 0.5 \\
\hline \multicolumn{9}{|l|}{ Gram (-) } \\
\hline Escherichiacoli ATCC 25922 & 1000 & 1000 & 250 & 500 & 1.9 & 1.9 & 0,5 & 1 \\
\hline E. coli-MQ11009 & 400 & 500 & 250 & 250 & 1 & 1 & 0,5 & 1 \\
\hline E. coli-MQ11068 & 500 & 500 & 250 & 500 & 1 & 1 & 1 & 1 \\
\hline E. coli-MQ11062 & 250 & 500 & 500 & 500 & 1 & 1 & 1 & 1.5 \\
\hline E. coli-MQ586 & 62.5 & 125 & 62.5 & 125 & 1 & 1 & 1.5 & 1.5 \\
\hline
\end{tabular}

MSSA: Methicillin-sensitive Staphylococcus aureus ATCC 25923; MRSA: Methicillin-resistant. S. aureus ATCC 43300; MIC: Minimum inhibitory concentration, MBC: Minimum Bactericidal Concentration.

Table 5. Combinatory effects of LdRe and LnRe with cefotaxime. The results are showed as MIC and MIC combination (MICcomb; expressed in $\mu \mathrm{g} / \mathrm{mL}$ ), fractional inhibitory concentration (FIC), fractional inhibitory concentration index (FICI) and dose reduction index (DRI) values.

\begin{tabular}{|c|c|c|c|c|c|c|c|c|c|c|c|c|}
\hline & \multicolumn{6}{|c|}{ MSSA } & \multicolumn{6}{|c|}{ MRSA } \\
\hline & MIC & MICcomb & FIC & FICI & DRI & Effect & MIC & MICcomb & FIC & FICI & DRI & Effect \\
\hline LdRe & 32 & 16 & 0.5 & & $?$ & Ind & 32 & 32 & 1 & & & \\
\hline Cef & 1 & 0.5 & 0.5 & 1 & 2 & Ind & 0.5 & 0.125 & 0.25 & 1.25 & 4 & Ind \\
\hline LnRe & 32 & 32 & 1 & & & & 16 & 8 & 0.5 & & & \\
\hline Cef & 1 & 0.25 & 0.25 & 1.25 & 4 & Ind & 0.5 & 0,25 & 0.5 & 1 & 2 & Ind \\
\hline
\end{tabular}

MSSA: Methicillin-sensitive Staphylococcus aureus ATCC 25923; MRSA: Methicillin-resistant S. aureus ATCC 43300; MIC: Minima Inhibitory Concentration; MICcomb: Minima Inhibitory Concentration combination; FIC: Fractional Inhibitory Concentration; FICI: Fractional Inhibitory Concentration Index; DRI: Dose Reduction Index; Ind: indifference. Synergy (FICI $\leq 0.5)$, no interaction or indifference (FICI > 0.5-4.0) and antagonism (FICI > 4).

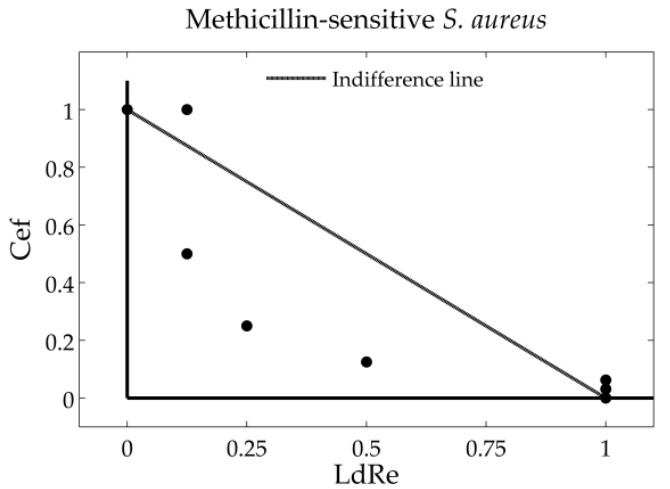

(A)

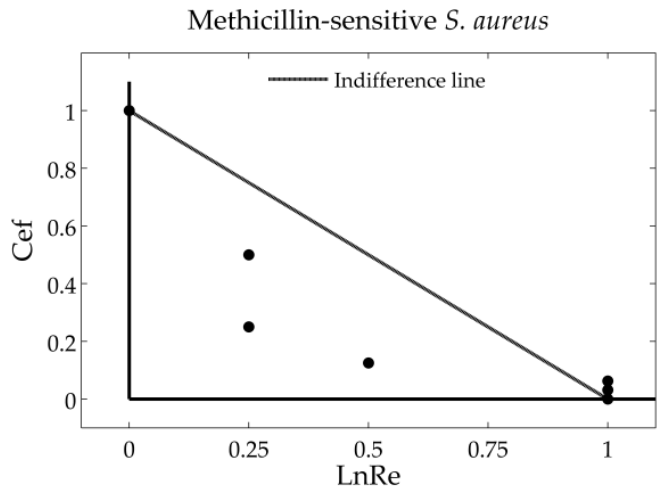

(B)

Figure 4. Cont. 


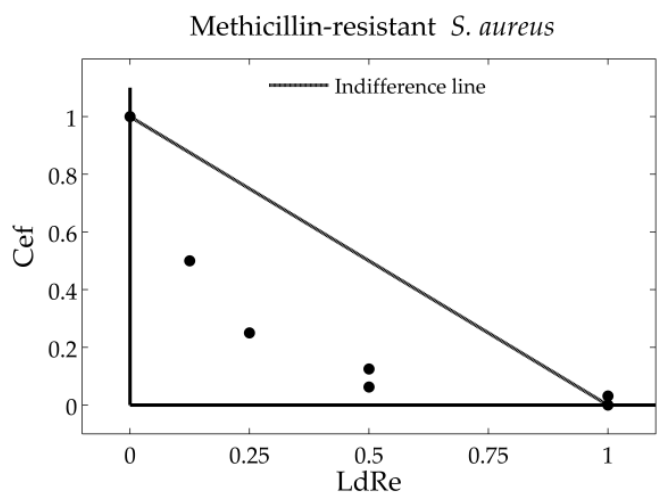

(C)

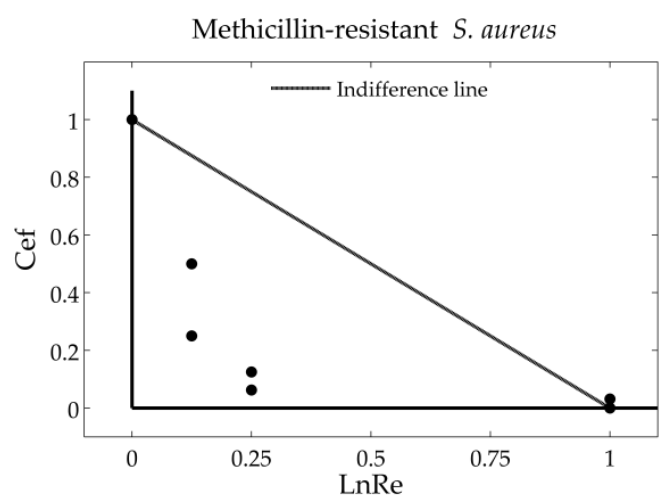

(D)

Figure 4. Normalized Isobolograms of LdRe or LnRe with cefotaxime against Methicillin-sensitive Staphylococcus aureus ATCC 25923 (MSSA) (A,B) and normalized Isobolograms of LdRe or LnRe with cefotaxime against Methicilin- resistant S. aureus ATCC 43300 (MRSA), respectively (C,D). The discontinuous line represents the indifference line and the points the experimental combinations at different levels.

The results represented in the isobologram (Figure 4) agreed with those obtained by the checkerboard analysis. The additive effect was found to both species tested (MSSA and MRSA) due to that the points the experimental combinations are below the line of indifference. Therefore, the combination between resins (LdRe and LnRe) could be an excellent strategy to reduce the drug doses, and thus, achieve the resistance bacteria.

The broad spectrum of biological activities such as antimicrobials, antioxidants, inhibitors of several enzymes, anti-inflammatory, antitumor and others, whichhave been reported for extracts and infusions of aerial parts of most species of this genus, have been regularly associated with the presence and content of phenolics or lignans and their derivatives [6-12]. In a previous work, the bioassay-guided isolation of L. nitida resin (LnRe) led to the isolation of $3^{\prime}$ methyl-nordihydroguaiaretic acid (MNDGA)20 and nordihydroguaiaretic acid (NDGA)13 and compound 19(4-[4-(4-hydroxy-phenyl)-2,3-dimethyl- butyl]-benzene1,2-diol) as the compounds mainly responsible for the antifungal activity. Additionally, two epoxylignans: meso-(rel $\left.7 \mathrm{~S}, 8 \mathrm{~S}, 7^{\prime} \mathrm{R}, 8^{\prime} \mathrm{R}\right)-3,4,3^{\prime}, 4^{\prime}$-tetrahydroxy- $7,7^{\prime}$-epoxylignan and compound $\mathbf{6}\left(7 \mathrm{~S}, 8 \mathrm{~S}, 7^{\prime} \mathrm{S}, 8^{\prime} \mathrm{S}\right)-3,3^{\prime}, 4^{\prime}$-trihy droxy-4-methoxy-7,7'-epoxylignan were isolated [26]. Recently the compounds 13,19 and 20 have been reported as marker compounds of synergistic mutual potentiation of antifungal activity of Zuccagnia punctata Cav. and L. nitida Cav. extracts in clinical isolates of Candida albicans and Candida glabrata. Additionally, in this previous work carried out in an Andean population in the center-west of Argentina, three marker compounds $(\mathbf{1 3}, 19$ and 20) for $L$ nitida were quantified over a year (February, May, September and November), highlighting their higher production in coincidence with the flowering period of the species, late spring and early summer (November and December) [11]. From the forty-one phenolics compounds detected and tentatively identified by UHPLC-ESI-OT-MS-MS in LdRe and LnRe resins, twenty-nine are lignans, mainly simple bisphenyl lignans and tricyclic lignans (cyclolignans and epoxylignans). The species belonging to the Larrea genus are rich in simple bisphenyl lignans and tricyclic lignans known as cyclolignans; those compounds support the pharmacological activities of extracts of this species as antiherpes, antioxidant, antifungal and anti-inflammatory. In the last decade, the strong activity of some lignans against the human immunodeficiency virus, human papilloma virus and cancer, their use in ameliorating neurodegenerative diseases and symptoms of aging has been reported. Additionally, molecular mechanisms underlying the antiviral and anticancer activities have been elucidated [40].

\section{Conclusions}

In this report, forty-one compounds were detected in LnRe resin by ultrahigh resolution liquid chromatography orbitrap MS analysis (UHPLC-PDA-OT-MS). From them, as far as we know, one phenolic compound, eight flavonoids and nineteen lignans are reported 
for the first time to this species, significantly updating the chemical composition of this interesting species. The results here reported support the recognized medicinal properties of these two recognized South American medicinal plants and on the other hand highlights them as a sustainable way of biomolecules of pharmacological interest. Additionally, relevant biological properties can be expected for these two species supported by the content and structural diversity mainly on the detection of bioactive lignans. The yellow-brown resins of the two Larrea showed a high content of TP, highlighting LnRe collected in the Andean mountains. This allows us to conclude that the resins from those wild species $L$ divaricata and $L$ nitida growing in Argentina represent a rich and sustainable source of antioxidants, mainly lignans. Additionally, the antibacterial activity of LdRe and LdRe showed a very strong activity against MSSA and MRSA. The combination between resins (LdRe and LnRe) with cefotaxime could be a potential strategy to reduce the drug doses, and in this way can be a potential alternative to reduce bacterial resistance. The new trend towards the use of natural extracts as pharmaceuticals rather than pure drugs opens a pathway for the development of a phytomedicine derived from resins of Larrea. This could be a promising pathway that needs to be intensively explored and developed in the near future.

Supplementary Materials: Are available online at https:/ / www.mdpi.com/2076-3921/10/2/185 /s1; Figure S1: Representative panel of Checkerboard design combining Larrea resins (LR) with Cefotaxime (Cef). GC stands for growth control. Dark circles show bacterial growth and white circles show no bacterial growth, Figure S2: Full orbitrap MS spectra and structures of representative compounds 13, 16, 20, 26 and 28 (a-e).

Author Contributions: M.J.S., B.L., G.E.F., and A.T. conceived and designed the experiments; J.G., S.M., M.P. and B.L., performed the antioxidant, nematicidal, antimicrobial, and total phenolics and flavonoids content experiments; M.J.S. and J.B. analyzed the data of HPLC/MS. All authors wrote the paper. All authors have read and agreed to the published version of the manuscript.

Funding: This research was funded by CICITCA-UNSJ, PME-2015-0200 Argentina. M.J.S. and J.B. received financial support from Fondecyt (Grant 1180059).

Institutional Review Board Statement: The lipoperoxidation in erythrocytes protocol assay was conducted in accordance with the Declaration of Helsinki, and is included in the approved project by CICIT-CA-UNSJ-Argentina (Project code 80020190100277SJ, Resolución N 0591-20-R-UNSJ) referred to the ethical and environmental safeguard, and to preserve the hygiene and safety conditions in the activities to be carried out in laboratories.

Informed Consent Statement: Informed consent was obtained from all subjects involved in the lipoperoxidation study.

Data Availability Statement: Not applicable.

Acknowledgments: J.G.: S.M. and M.P. held fellowships from CONICET, Argentina. G.E.F. and B.L. are researchers from CONICET, Argentina.

Conflicts of Interest: The authors do not have any conflict of interest.

\section{References}

1. Licá, I.C.; Dos Santos Soares, A.M.; Silva de Mesquita, L.S.; Malik, S. Biological properties and pharmacological potential of plant exudates. Food Res. Int. 2018, 105, 1039-1053. [CrossRef] [PubMed]

2. Feresin, G.E.; Tapia, A.; Gimenez, A.; Ravelo, A.G.; Zacchino, S.A.; Sortino, M.; Schmeda-Hirschmann, G. Constituents of the Argentinian medicinal plant Baccharis grisebachii and their antimicrobial activity. J. Ethnopharmacol. 2003, 89, 73-80. [CrossRef]

3. Tapia, A.; Rodriguez, J.; Theoduloz, C.; Lopez, S.; Feresin, G.E.; Schmeda-Hirschmann, G. Free radical scavengers and antioxidants from Baccharis grisebachii. J. Ethnopharmacol. 2004, 95, 155-161. [CrossRef] [PubMed]

4. Modak, B.; Salina, M.; Rodilla, J.M.L.; Torres, R. Study of the chemical composition of the resinous exudate isolated from Heliotropium sclerocarpum and evaluation of the antioxidant properties of the phenolic compounds and the resin. Molecules 2009, 14, 4625-4633. [CrossRef]

5. Gómez, J.; Simirgiotis, M.; Manrique, S.; Lima, B.; Bórquez, J.; Feresin, G.E.; Tapia, A. UHPLC-HESI-OT-MS-MS biomolecules profiling, antioxidant and antibacterial activity of the "orange-yellow resin" from Zuccagnia punctata cav. Antioxidants 2020, 9, 123. [CrossRef]

6. Kiesling, R. Flora de San Juan. República Argentina; Estudio Sigma: Valladolid, Spain, 2003; Volumen II. 
7. The Plant List. Available online: http:/ / www.theplantlist.org/tpl1.1/search?q=larrea (accessed on 20 November 2020).

8. Anesini, C.; Ferraro, G.; López, P.; Borda, E. Different intracelular signals coupled to the antiproliferative action of aqueous crude extract from Larrea divaricata Cav. and nor-dihydroguaiaretic acid on a lymphoma cell line. Phytomedicine 2001, 8, 1-7. [CrossRef]

9. Bongiovanni, G.; Cantero, J.; Eynard, A.; Goleniowski, M. Organic extracts of Larrea divaricata Cav. induced apoptosis on tumoral MCF7 cells with an higher citotoxicity than nordihydroguaiaretic acid or Paclitaxel. J. Exp. Ther. Oncol. $2008,7,1-7$.

10. Martins, S.; Amorim, E.L.C.; da Silva Peixoto Sobrinho, T.J.; Saraiva, A.M.; Pisciottano, M.N.; Aguilar, C.N.; Teixeira, J.A.; Mussatto, S.I. Antibacterial activity of crude methanolic extract and fractions obtained from Larrea tridentata leaves. Ind. Crop. Prod. 2013, 41, 306-311. [CrossRef]

11. Butassi, E.; Svetaz, L.; Ivancovich, J.J.; Feresin, G.; Tapia, A.; Zacchino, S. Synergistic antifungal fixed-ratio combinations of Zuccagnia punctata Cav. and Larrea nitida Cav., using mixed-effects loewe (MixLow) method. Phytomedicine 2015, 22, 666-678. [CrossRef]

12. Peralta, I.; Marrassini, C.; Filip, R.; Alonso, M.R.; Anesini, C. Food preservation by Larrea divaricata extract: Participation of polyphenols. Food Sci. Nutr. 2018, 6, 1269-1275. [CrossRef]

13. Skouta, R.; Morán-Santibañez, K.; Valenzuela, C.A.; Vasquez, A.H.; Fenelon, K. Assessing the antioxidant properties of Larrea tridentata extract as a potential molecular therapy against oxidative stress. Molecules 2018, 23, 1826. [CrossRef] [PubMed]

14. Peralta, I.; Marrassini, C.; Barreiro Arcos, M.L.; Cremaschi, G.A.; Alonso, M.R.; Anesini, C. Larrea divaricata Cav. Aqueous extract and nordihydroguariaretic acid modulate oxidative stress in submandibular glands of diabetic rats: A buccal protective in diabetes. BMC Complement. Altern. Med. 2019, 19, 227. [CrossRef] [PubMed]

15. Butassi, E.; Svetaz, L.A.; Sortino, M.A.; Quiroga, A.D.; Carvalho, V.S.; Cortés, J.C.G.; Ribas, J.C.; Zacchino, S.A. Approaches to the mechanism of antifungal activity of Zuccagnia punctata-Larrea nitida bi-herbal combination. Phytomedicine 2019, 54, $291-301$. [CrossRef]

16. Sagaste, C.A.; Montero, G.; Coronado, M.A.; Ayala, J.R.; León, J.A.; García, C.; Rojano, B.A.; Rosales, S.; Montes, D.G. Creosote Bush (Larrea tridentata) extract assessment as a green antioxidant for biodiesel. Molecules 2019, 24, 1786. [CrossRef] [PubMed]

17. Morán-Santibañez, K.; Vasquez, A.H.; Varela-Ramirez, A.; Henderson, V.; Sweeney, J.; Odero-Marah, V.; Fenelon, K.; Skouta, R. Larrea tridentata extract mitigates oxidative stress-induced cytotoxicity in human neuroblastoma SH-SY5Y cells. Antioxidants 2019, 8, 427. [CrossRef]

18. Moreno, M.A.; Bojorges, H.; Falco, I.; Sanchez, G.; Lopez-Carballo, G.; Lopez-Rubio, A.; Zampini, I.C.; Isla, M.I.; Fabra, M.J. Active properties of edible marine polysaccharide-based coatings containing Larrea nitida polyphenols enriched extract. Food Hydrocoll. 2020, 102, 105595. [CrossRef]

19. Quispe, C.; Villalobos, M.; Borquez, J.; Simirgiotis, M. Chemical composition and antioxidant activity of aloe vera from the Pica Oasis (Tarapacá, Chile) by UHPLC-Q/Orbitrap/MS/MS. J. Chem. 2018, 2018, 1-12. [CrossRef]

20. Simirgiotis, M.J.; Quispe, C.; Mocan, A.; Villatoro, J.M.; Areche, C.; Bórquez, J.; Sepúlveda, B.; Echiburu-Chau, C. UHPLC high resolution orbitrap metabolomic fingerprinting of the unique species Ophryosporus triangularismeyen from the atacama desert, Northern Chile. Rev. Bras. Farmacogn. 2017, 27, 179-187. [CrossRef]

21. Luna, L.; Simirgiotis, M.; Lima, B.; Bórquez, J.; Feresin, G.E.; Tapia, A. UHPLC-MS metabolome fingerprinting: The isolation of main compounds and antioxidant activity of the andean species Tetraglochin ameghinoi (Speg.) speg. Molecules 2018, $23,793$. [CrossRef]

22. Simirgiotis, M.; Quispe, C.; Areche, C.; Sepulveda, B. Phenolic compounds in chilean mistletoe (Quintral, Tristerix tetrandus) analyzed by UHPLC-Q/Orbitrap/MS/MS and its antioxidant properties. Molecules 2016, 21, 245. [CrossRef]

23. Simirgiotis, M.J.; Quispe, C.; Bórquez, J.; Schmeda-Hirschmann, G.; Avendaño, M.; Sepúlveda, B.; Winterhalter, P. Fast high resolution Orbitrap MS fingerprinting of the resin of Heliotropium taltalense Phil. from the Atacama Desert. Ind. Crop. Prod. 2016, 85, 159-166. [CrossRef]

24. Gómez, J.; Simirgiotis, M.; Lima, B.; Gamarra-Luques, C.; Bórquez, J.; Caballero, D.; Feresin, G.E.; Tapia, A. UHPLCQ/Orbitrap/MS/MS fingerprinting, free radical scavenging, and antimicrobial activity of Tessaria absinthiodes (Hook. \&Arn.) DC. (Asteraceae) Lyophilized decoction from Argentina and Chile. Antioxidants 2019, 8, 593. [CrossRef]

25. Cifuentes, F.; Palacios, J.; Nwokocha, C.; Bórquez, J.; Simirgiotis, M.; Norambuena, I.; Chiong, M.; Paredes, A. Polyphenolic composition and hypotensive effects of Parastrephia quadrangularis (Meyen) cabrera in rat. Antioxidants 2019, 8, 591. [CrossRef] [PubMed]

26. Agüero, M.B.; Svetaz, L.; Sánchez, M.; Luna, L.; Lima, B.; López, M.L.; Zacchino, S.; Palermo, J.A.; Wunderlin, D.; Feresin, G.E.; et al. Argentinean propolis from Larrea nitida Cav. (Zygophyllaceae) exudates: Phytochemical characterization and antifungal activity. Food Chem. Toxicol. 2011, 49, 1970-1978.

27. Aissani, N.; Urgeghe, P.P.; Oplos, C.; Saba, M.; Tocco, G.; Petretto, G.L.; Eloh, K.; Menkissoglu-Spiroudi, U.; Ntalli, N.; Caboni, P. Nematicidal activity of the volatilome of eruca sativa on Meloidogyne incognita. J. Agric. Food Chem. 2015, 63, 6120-6125. [CrossRef]

28. Clinical and Laboratory Standards Institute. Performance Standards for Antimicrobial Susceptibility Testing; Twenty-Third Informational Supplement; CLSI document M100-S23; Clinical and Laboratory Standards Institute: Wayne, PA, USA, 2013; ISBN 1-56238-865-7.

29. Hemaiswarya, S.; Kruthiventi, A.K.; Doble, M. Synergism between natural products and antibiotics against infectious diseases. Phytomedicine 2008, 15, 639-652. [CrossRef] 
30. Odds, F.C. Synergy, antagonism, and what the chequerboard puts between them. J. Antimicrob. Chemother. 2003, 52, 1. [CrossRef]

31. Chou, T.C. Theoretical basis, experimental design, and computerized simulation of synergism and antagonism in drug combination studies. Pharmacol. Rev. 2006, 58, 621-681. [CrossRef]

32. Gessner, P.K. Isobolographic analysis of interactions: An update on applications and utility. Toxicology 1995, 105, 161-179. [CrossRef]

33. Gezginci, M.H.; Timmermann, B.N. A short synthetic route to nordihydroguaiaretic acid (NDGA) and its stereoisomer using ti-induced carbonyl-coupling reaction. Tetrahedron. Lett. 2001, 42, 6083-6085. [CrossRef]

34. Gisvold, O.; Thaker, E. Lignans from Larrea divaricata. J. Pharm. Sci. 1974, 63, 1905-1907. [CrossRef] [PubMed]

35. Torres, R.; Urzúa, A.; Modak, B. Isopregomisin, a 1, 4-Bis(phenyl)-2, 3-dimethylbutane Lignan from Porlieria chilensis. J. Nat. Prod. 1989, 52, 402-403. [CrossRef]

36. Torres, R.; Urbina, F.; Morales, C.; Modak, B.; Monache, F.D. Antioxidant properties of lignans and ferulic acid from the resinous exudate of Larrea nitida. J. Chil. Chem. Soc. 2003, 48, 61-63. [CrossRef]

37. Jeong, H.; Kim, S.; Lee, J.; Park, J.Y.; Zhou, W.; Liu, X.; Kim, S.D.; Song, Y.S.; Jang, C.Y.; Oh, S.R.; et al. Characterization of phase I and phase II hepatic metabolism and reactive intermediates of Larrea nitida Cav. and its lignan compounds. Phytother. Res. 2017, 31, 140-151. [CrossRef] [PubMed]

38. Gnabre, J.; Huang, R.C.C.; Bates, R.B.; Burns, J.J.; Caldera, S.; Malcomson, M.E.; McClure, K.J. Characterization of Anti-HIV lignans from Larrea tridentate. Tetrahedron 1995, 51, 12203-12210. [CrossRef]

39. Moreno, M.A.; Córdoba, S.; Zampini, I.C.; Mercado, M.I.; Ponessa, G.; Sayago, J.E.; Pino Ramos, L.L.; Schmeda-Hirschmann, G.; Isla, M.I. Argentinean larrea dry extracts with potential use in vaginal candidiasis. Nat. Prod. Commun. 2018, 13, 171-174. [CrossRef]

40. Gnabre, J.; Bates, R.; Huang, R.C. Creosote bush lignans for human disease treatment and prevention: Perspectives on combination therapy. J. Tradit. Complement. Med. 2015, 5, 119-126. [CrossRef]

41. Gnabre, J.N.; Ito, Y.; Ma, Y.; Huang, R.C. Isolation of anti-HIV-1 lignans from Larrea tridentata by counter-current chroma-tography. J. Chromatogr. A 1996, 719, 353-364. [CrossRef]

42. Sakakibara, M.; Difeo, D.; Nakatani, N.; Timmermann, B.; Mabry, T.J. Flavonoid methyl ethers on the external leaf surface of Larrea tridentata and L. divaricata. Phytochemistry 1976, 15, 727-731. [CrossRef]

43. Chitwood, D.J. Phytochemicals based strategies for nematode control. Ann. Rev. Phytopathol. 2002, 40, 221-249. [CrossRef]

44. Ríos, J.L.; Recio, M.C. Medicinal plants and antimicrobial activity. J. Ethnopharmacol. 2005, 100, 80-84. [CrossRef] [PubMed]

45. Abu El-Wafa, W.M.; Ahmed, R.H.; Abdel-Halim Ramadan, M. Synergistic effects of pomegranate and rosemary extracts in combination with antibiotics against antibiotic resistance and biofilm formation of Pseudomonas aeruginosa. Braz. J. Microbiol. 2020, 51, 1079-1092. [CrossRef] [PubMed]

46. Gorlenko, C.L.; Yu, K.H.; Budanova, E.V.; Zamyatnin, A.A., Jr.; Ikryannikova, L.N. Plant secondary metabolites in the battle of drugs and drug-resistant bacteria: New heroes or worse clones of antibiotics? Antibiotics 2020, 9, 170. [CrossRef] [PubMed]

47. Zacchino, S.A.; Butassi, E.; Di Liberto, M.; Raimondi, M.; Postigo, A.; Sortino, M. Review Plant phenolics and terpenoids as adjuvants of antibacterial and antifungal drugs. Phytomedicine 2017, 37, 27-48. [CrossRef]

48. Khameneh, B.; Iranshahy, M.; Soheili, V.; FazlyBazzaz, B.S. Review on plant antimicrobials: A mechanistic viewpoint. Antimicrob. Resist. Infect. Control. 2019, 8, 1-28. [CrossRef] 